\title{
Preoperative Serum Albumin Level As A Predictor Of Postoperative Pneumonia After Femoral Neck Fracture Surgery In A Geriatric Population
}

This article was published in the following Dove Press journal: Clinical Interventions in Aging

\author{
Yakang Wang' \\ Xiaoli $\mathrm{Li}^{2}$ \\ Yahong $\mathrm{Ji}^{2}$ \\ Hua Tian $^{2}$ \\ Xiaofang Liang ${ }^{2}$ \\ $\mathrm{Na} \mathrm{Li}{ }^{2}$ \\ Junning Wang $\mathbb{D}^{2}$
}

'Department of Joint Surgery, Honghui Hospital, Xi'an Jiaotong University, X'an, Shaanxi 710054, People's Republic of China; ${ }^{2}$ Department of Respiratory and Gastroenterology, Honghui Hospital, Xi'an Jiaotong University, Xi'an, Shaanxi 710054, People's Republic of China
Correspondence: Junning Wang Department of Respiratory and Gastroenterology, Honghui Hospital, Xi'an Jiaotong University, No. 555, Youyi East Road, Nanshaomen, Xi'an, Shaanxi 710054, People's Republic of China $\mathrm{Tel} / \mathrm{Fax}+862962818354$

Email wangjunningde@।63.com
Purpose: Femoral neck fracture usually occurs in the geriatric population. Postoperative pneumonia (POP) is known to be devastated, and it is the most frequent complication among patients receiving surgical treatment for femoral neck fractures. However, whether patients who have hypoalbuminaemia are susceptible to the development of POP is a serious concern, although it has not been investigated. We attempted to investigate the association between newly developed POP and hypoalbuminaemia and to identify whether hypoalbuminaemia is an independent risk factor for POP after femoral neck fracture in geriatric population.

Patients and methods: We retrospectively reviewed the records from the first 30 days after surgery of patients who were $\geq 65$ years of age and who had a femoral neck fracture treated with surgery between January 2018 and December 2018 at the Honghui Hospital, $\mathrm{Xi}$ 'an Jiaotong University. Patients were divided into two groups based on whether they did or did not experience POP, and their clinical characteristics were compared. Binomial logistic regression was used to identify potential risk factors of POP by analysing demographic factors, preoperative comorbidities, laboratory results, and surgical factors.

Results: A total of 720 patients were included in the analysis, and 54 patients experienced POP. The incidence of POP after surgical treatment for a femoral neck fracture in this geriatric population was $7.5 \%$. In addition, patients with POP had significantly longer hospital stays than patients without POP. The binary logistic regression analysis revealed that preoperative hypoalbuminaemia [odds ratio $=5.187,95 \%$ confidence interval (CI): 2.561-10.506, $\mathrm{P}<0.0001$ ], COPD ( $\mathrm{OR}=3.819,95 \% \mathrm{CI}: 1.247-11.701, \mathrm{P}=0.019)$, prior stroke $(\mathrm{OR}=3.107,95 \% \mathrm{CI}: 1.470-6.568, \mathrm{P}=0.003)$ and the time from injury to surgery (OR $=1.076$, 95\% CI: $1.034-1.119, \mathrm{P}<0.0001)$ were predominant and independent risk factors associated with POP after femoral neck fracture in this geriatric population.

Conclusion: The current study revealed that among a geriatric population admitted for femoral neck fracture surgery, preoperative hypoalbuminaemia was a predictor of POP, followed by COPD, prior stroke and the time from injury to surgery. Thus, patients who undergo femoral neck fracture surgery and have preoperative hypoalbuminaemia should receive additional monitoring and perioperative care.

Keywords: femoral neck fracture, nutrients, serum albumin, hypoalbuminaemia, postoperative pneumonia, risk factor

\section{Introduction}

Femoral neck fractures usually occur following falls, which are very common in the geriatric population, accounting for $48.22 \%$ of hip fractures and $3.13 \%$ of all fractures in adults. ${ }^{1}$ With ageing populations, an increase in the number of femoral neck 
fractures is inevitable; age-associated low bone mineral density, malnutrition and accompanying vitamin depletion, as well as the presence of other pre-existing risk factors, makes the geriatric population especially susceptible to fracture. There are a number of treatment options for fractures of the femoral neck, including hemiarthroplasty (HA), arthroplasty (THA), internal fixation and non-operative treatment. Surgery to treat femoral neck fractures is considered the gold standard for elderly patients to restore the normal range of motion in order to perform activities of daily living. ${ }^{2}$ The degree of mortality associated with a femoral neck fracture is reported to be from $8.4 \%$ to $36 \%$ 1 year after surgical repair. ${ }^{3}$ Most deaths occur because geriatric patients have underlying comorbidities and postoperative complications. Among all the postoperative complications, postoperative pneumonia (POP) is known to be devastated, and it is the most frequent complication in the femoral neck fracture population. ${ }^{3-5}$ Specifically, authors have shown that POP affects from $4 \%$ to $9 \%$ of all femoral neck fracture patients. ${ }^{3,5,6}$ In clinical practice, despite standard therapy and intensive monitoring, patients with POP are associated with an increased surgical mortality rate, increased in-hospital cost, and prolonged length of hospital stay, and POP influences the recovery of patients' functional status. At one time, the 30-day mortality rate of femoral neck fracture patients with POP was reported to be as high as $14 \%$ compared to only $1.7 \%$ for those without POP. ${ }^{6}$ Many published studies have shown that POP is usually related to the preoperative status of the patient. Prior studies have identified numerous patient-related or procedure-related risk factors for POP after femoral neck fracture surgery. In most but not all studies, multiple variables, including older age, male sex, and the presence of several key clinical comorbidities such as chronic obstructive pulmonary disease (COPD), ${ }^{5}$ obesity, ${ }^{7,8}$ tobacco smoking, ${ }^{9}$ prior stroke, ${ }^{3}$ impaired preoperative pulmonary function, ${ }^{10,11}$ cognitive and functional premorbid status ${ }^{12}$ and cardiovascular disease ${ }^{3}$ have been identified as risk factors for POP.

Preoperative malnutrition status has been identified as another potential risk factor and prognostic predictor for poor outcomes following hip fracture surgery. ${ }^{13}$ Serum albumin level is the conventional indicator used as a biomarker of malnutrition, and patients are considered to have poor nutritional status when their serum albumin level is $<35 \mathrm{~g} / \mathrm{L},{ }^{14}$ Several previous studies have indicated that preoperative serum albumin indicates susceptibility to the development of postoperative complications in patients with malignancies, including periampullary tumours, ${ }^{15}$ colorectal cancer, ${ }^{16}$ and lung cancer. ${ }^{17}$ Likewise, in noncancer patients, the relationships between hypoalbuminaemia and poor postoperative outcomes have been studied. For example, preoperative hypoalbuminaemia has been identified as a risk factor for delayed wound healing and surgical-site infection after total joint arthroplasty. ${ }^{18}$ However, few report shave focused on preoperative albumin levels contributing to POP risk following femoral neck fracture surgery.

We aimed to investigate the relationship between preoperative albumin levels and postoperative pneumonia and to identify whether hypoalbuminaemia is an independent risk factor for POP in geriatric population who underwent surgical treatment for femoral neck fracture in our hospital. The results may help to stratify risk for patients and provide guidance for clinicians to determine appropriate therapeutic strategies.

\section{Materials And Methods Study Population}

This was a retrospective evaluation of the clinical records of all geriatric patients who were $\geq 65$ years of age and had received surgical treatment for acute femoral neck fracture from recent falls at the Honghui Hospital (Xi'an Jiaotong University, Shannxi, China), a tertiary-care teaching hospital, between January 2018 and December 2018. We reviewed 824 patients with inclusion and exclusion criteria. Finally, a total of 720 patients were identified as eligible for enrolment in the study; 54 subjects were diagnosed with POP $(7.5 \%, 54$ out of 720$)$, and the remaining 666 subjects were placed in the non-POP group (Figure 1). The inclusion criteria for this study were an age of 65 years or older and diagnosis and confirmation of a femoral neck fracture using X-ray and/or computed tomography. The exclusion criteria included patients who (1) did not undergo the required surgical intervention, (2) had X-ray and/or computed tomography-confirmed preoperative pneumonia, (3) had multiple bone fractures, (4) had incomplete data or their data could not be obtained, (5) were lost to follow-up at one month, and (6) who had pathological fractures. According to their clinical characteristics, patients were divided into POP and non-POP groups. The current study protocol was approved by the Ethics Committee of Honghui Hospital, Xi'an Jiaotong University (No. 201903004) and complied with the principium plan in the Helsinki Declaration. The requirement 


\section{Patients who were $\geq 65$ years of age with hospitalization for femoral neck fracture from January 2018 to December 2018 at the Honghui Hospital, Xi'an Jiaotong University}

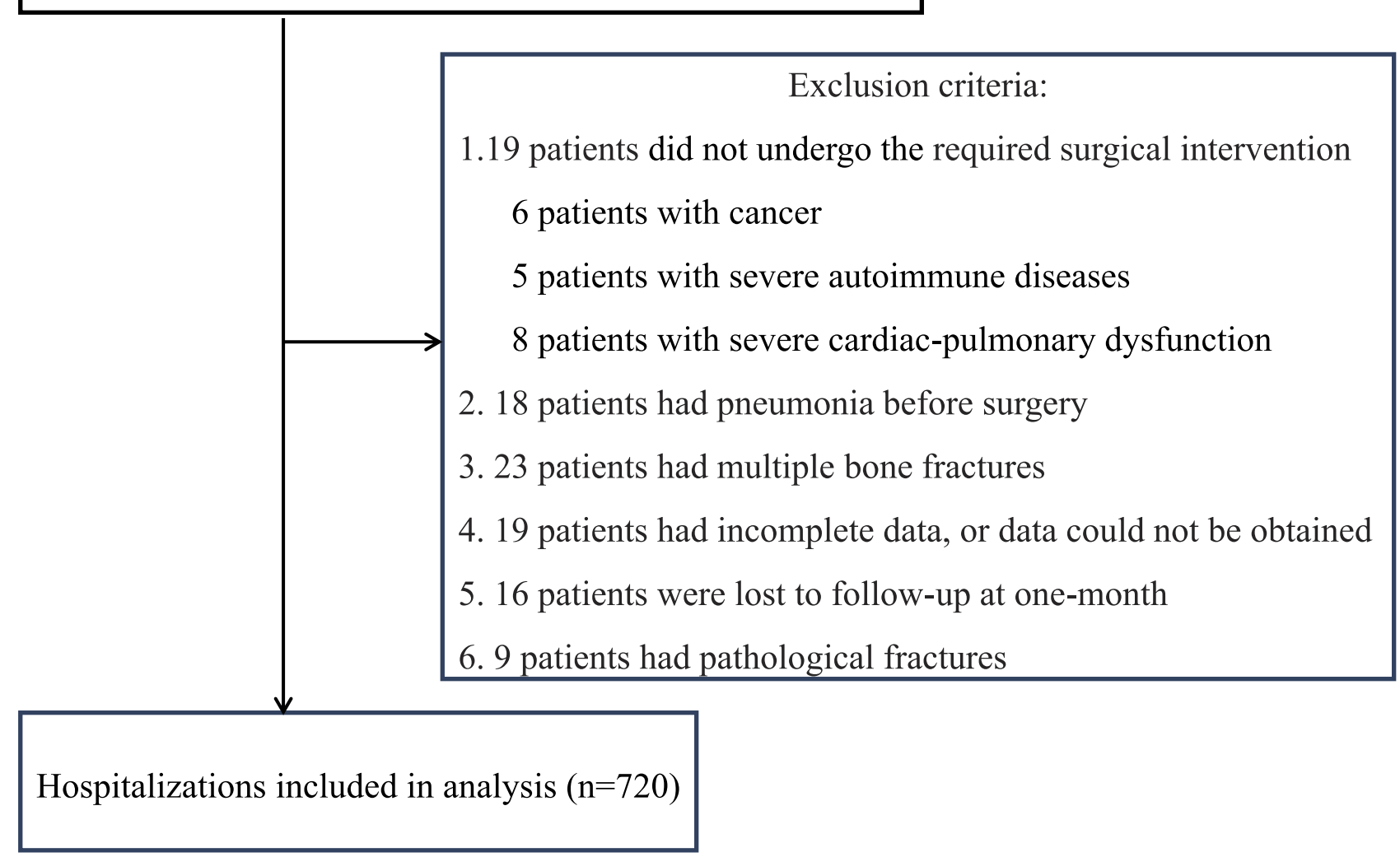

Figure I A flowchart shows the patient selection process.

for written informed consent was waived because this was a retrospective analysis. The information from patient records was anonymized. Highly trained nurses performed the clinical reviews and then followed up with the patients during the first 30 postoperative days to track the occurrence of postoperative adverse events.

\section{Data Collection}

For the present study, demographic features, preoperative comorbidities, laboratory test results, and surgical factors were all recorded. Demographic data included age, sex, height, and weight. Major comorbidities included COPD, type 2 diabetes, circulatory abnormalities (hypertension, coronary heart disease, and arrhythmia), and prior stroke. For a better description of the patients' respiratory system status, their history of smoking was also recorded. Routine biochemical analyses of patients' blood, in addition to serum albumin concentration, other laboratory analyses, including those for total bilirubin, haemoglobin, blood urea nitrogen, creatinine, glutamic oxaloacetic transaminase (AST) and glutamic pyruvic transaminase (ALT), were performed for each patient in the morning the next day of admission to our hospital. At the time of admission, all patients had chest X-rays performed before surgery. The treatment details, including the surgical type, the duration of the surgery, the intraoperative blood loss, and the length of hospital stay, were also studied. Whether pneumonia was a complication after surgery, the data from the surgical operation and the patients' clinical characteristics were recorded in a database. Finally, patients without preoperative serum albumin laboratory results or patients with missing preoperative data of interest were excluded. For the purposes of the outcome analyses and consistent with previous studies, serum albumin levels 
were stratified as follows: hypoalbuminaemia (albumin $<35 \mathrm{~g} / \mathrm{L}$ ) and normal albumin (albumin $\geq 3.5 \mathrm{~g} / \mathrm{L}$ ); clinical data were collected for the entire 30-day postoperative period.

\section{Diagnosis Of Postoperative Pneumonia (POP)}

The primary outcome for this study was the incidence of $\mathrm{POP}$ occurring within the first 30 days postoperatively. Patients with POP were defined as those who did not have preoperative pneumonia and who had new or increasing lung infiltrations on postoperative chest X-ray and/or computed tomography images. Patients were considered to present without POP until one or more of the following criteria were met: (1) presence of new and/or progressive and steady respiratory symptoms, including purulent secretions and coughing; (2) presence of fever (fever with a body temperature of $>38^{\circ} \mathrm{C}$ ) or hypothermia (body temperature $<36.0^{\circ} \mathrm{C}$ ); (3) physical examination with signs of lung consolidation and/or moist rale; (4) lab test indicated leucocytosis (a white cell count of greater than $10 \times 10^{9} / \mathrm{L}$ ) or leukopenia (a white cell count of less than $4 \times 10^{9} / \mathrm{L}$ ); and (5) microbiological culture of sputum samples or blood samples indicating POP. ${ }^{3,19}$

\section{Statistical Analysis}

Patients were divided into two groups: a POP group and a non-POP group. A statistical analysis of the descriptive characteristics of the entire cohort was performed before subsequent analysis. Continuous, normally distributed quantitative data are reported as the mean \pm standard deviation (SD), and non-normal quantitative variables are reported as medians with interquartile ranges (IQRs) (25th and 75th percentiles). Clinical features were compared between the two groups. Normally distributed continuous variables were analysed via the Student's t-test, and the non-normal data were tested with the Mann-Whitney U-test. To study the categorical variables, the number of patients in each category was counted and recorded as a count (percentage), and the between-groups differences were compared using univariate analysis by Fisher's exact tests or Pearson chi-square tests ( $\chi^{2}$ test) of independence. Binomial logistic regression analysis with the forward stepwise conditional method was conducted to identify potential risk factors for POP and to determine the associations between serum albumin level and POP in the femoral neck fracture population. First, univariate analysis was utilized to assess potential risk factors for $\mathrm{POP}$, and then variables with $\mathrm{P}$ values $<0.1$ were used in the adjusted multivariable analysis. The outcomes of the regression analysis were recorded as odds ratios (ORs) and $95 \%$ confidence intervals $(95 \% \mathrm{CI})$. All tests were twosided, and the level of significance was set at $p<0.05$. All statistical analyses were conducted using SPSS (IBM SPSS Statistics, version 19.0).

\section{Results}

\section{The Clinical Characteristics Of The POP And Non-POP Groups}

The demographic, comorbidity, and laboratory characteristics of the 720 patients who underwent surgical treatment for femoral neck fracture and who were identified as eligible for participation in this study are shown in Table 1. A total of 54 subjects were diagnosed with POP $(7.5 \%, 54$ out of 720 patients), and the remaining 666 patients were placed in the non-POP group. A total of 125 patients $(17.36 \%, 125$ in 720$)$ with a preoperative albumin level less than $35 \mathrm{~g} / \mathrm{L}$ were identified as having hypoalbuminaemia. On preoperative evaluation, the average albumin concentration of the POP group was significantly lower than that of the non-POP group (33.9 \pm 4.7 vs $38.7 \pm 3.7, \mathrm{P}<0.0001$ ), and more patients had hypoalbuminaemia in the POP group than in the non-POP group [32 (59.25\%) vs 139 (20.87\%), $\mathrm{P}<0.0001]$. Admission haemoglobin was lower in the POP group than in the non-POP group $(112 \pm 13$ vs $121 \pm 21, P=0.001)$. Serum creatinine values were higher in the POP group than in the non-POP group ( $81 \pm 35 \mathrm{vs}$ $67 \pm 21, \mathrm{P}<0.0001$ ). Unexpectedly, BUN, ALT, AST, and TBIL were not associated with the occurrence of POP. Compared with the non-POP patients, patients with POP were significantly older (77.5 \pm 8.5 vs $82.3 \pm 8.1, \mathrm{P}<0.0001)$. Unexpectedly, sex and BMI were not associated with the occurrence of POP. Patients in the POP group tended to have a higher rate of comorbidities, including history of smoking [15 (27.8\%) vs 89 (13.3\%), P $=0.004]$, COPD [14 (25.9\%) vs $33(4.9 \%)$, $\mathrm{P}<0.0001]$ and prior stroke[17 (31.4\%) vs $112(16.8 \%)$, $\mathrm{P}=0.007]$, than patients in the non-POP group. There was no difference regarding circulatory comorbidities (hypertension, coronary heart disease and arrhythmia), diabetes history, or surgical parameters. Furthermore, patients with POP had a longer time from injury to surgery than those without POP (13.4 \pm 8.9 vs $6.8 \pm 5.4, \mathrm{P}<0.0001$ ), and the length of hospital stay in the group with POP was longer than that in the group without POP $(15.1 \pm 13.1$ vs $9.3 \pm 3.4, \mathrm{P}<0.0001)$. The selection of surgical approaches (hemiarthroplasty, arthroplasty, or 
Table I Comparison Of The Clinical Characteristics Of The POP And Non-POP Groups

\begin{tabular}{|c|c|c|c|c|}
\hline Items & POP (N =54) & Non-POP $(N=666)$ & $\chi^{2}$ Or t-value & $P$ value \\
\hline Age (years) & $82.3 \pm 8.1$ & $77.5 \pm 8.5$ & 3.97 & $<0.0001$ \\
\hline Sex, men/women & $20 / 34$ & $273 / 393$ & 0.324 & 0.569 \\
\hline BMI $\left(\mathrm{kg} / \mathrm{m}^{2}\right)$ & $22.1 \pm 2.3$ & $22.3 \pm 1.9$ & 1.23 & 0.219 \\
\hline History of smoking (\%) & $15(27.8 \%)$ & 89 (I3.3\%) & 8.397 & 0.004 \\
\hline COPD (\%) & 14 (25.9\%) & $33(4.9 \%)$ & 48.18 & $<0.0001$ \\
\hline Type 2 diabetes (\%) & $16(29.6 \%)$ & $132(19.8 \%)$ & 2.94 & 0.086 \\
\hline \multicolumn{5}{|l|}{ Circulatory diseases } \\
\hline Arrhythmia (\%) & $20(37 \%)$ & 189 (28.4\%) & 1.818 & 0.178 \\
\hline Coronary artery disease (\%) & $26(48.1 \%)$ & 294 (44.1\%) & 0.324 & 0.569 \\
\hline Hypertension (\%) & $29(53.7 \%)$ & $3 I I(46.7 \%)$ & 0.32 & 0.571 \\
\hline Prior stroke (\%) & 17 (3I.4\%) & 112 (I6.8\%) & 7.304 & 0.007 \\
\hline Hypoalbuminaemia (\%) & $32(59.25 \%)$ & 93 (13.96\%) & 71.43 & $<0.0001$ \\
\hline Alb $(g / L)$ & $33.9 \pm 4.7$ & $38.7 \pm 3.7$ & 8.72 & $<0.0001$ \\
\hline $\mathrm{Hb}(\mathrm{g} / \mathrm{L})$ & $112 \pm 13$ & $|2| \pm 2 \mid$ & 3.355 & 0.001 \\
\hline $\mathrm{Cr}(\mu \mathrm{mol} / \mathrm{L})$ & $81 \pm 35$ & $67 \pm 21$ & 4.4 & $<0.0001$ \\
\hline BUN (mmol/L) & $6.5 \pm 2.5$ & $6.2 \pm 2.8$ & 0.67 & 0.49 \\
\hline $\mathrm{ALT}(\mathrm{U} / \mathrm{L})$ & $21.5 \pm 24.5$ & $\mid 8.1 \pm 20.3$ & 1.115 & 0.249 \\
\hline AST (U/L) & $18.24 \pm 8.2$ & $20.4 \pm 17.6$ & 0.931 & 0.352 \\
\hline TBIL (umol/L) & $16.8 \pm 11.2$ & $18.2 \pm 1 \mid .5$ & 0.867 & 0.386 \\
\hline Surgical duration (mins) & $77.6 \pm 27.1$ & $81.9 \pm 26.3$ & 1.171 & 0.242 \\
\hline Blood loss $(\mathrm{mL})$ & $219.3 \pm \mid 14.1$ & $222.3 \pm 123.1$ & 0.179 & 0.858 \\
\hline Time from injury to surgery (d) & $13.4 \pm 8.9$ & $6.8 \pm 5.4$ & 8.124 & $<0.0001$ \\
\hline Length of hospital stay (d) & $15.1 \pm 13.1$ & $9.3 \pm 3.4$ & 8.295 & $<0.0001$ \\
\hline Surgical approaches & & & 3.324 & 0.190 \\
\hline Total hip arthroplasty (\%) & $6(11.1 \%)$ & 143 (2I.4\%) & & \\
\hline Intramedullary fixation (\%) & 8 (I4.8\%) & $94(14.1 \%)$ & & \\
\hline Half hip replacement (\%) & 40 (74. I\%) & $429(64.5 \%)$ & & \\
\hline
\end{tabular}

Notes: The data are presented as percentage $(\mathrm{N})$ for categorical variables, mean \pm SD for continuous variables (normal distribution), median (IQR) for continuous variables (non-normal distribution).

Abbreviations: POP, postoperative pneumonia; BMI, body mass index; COPD, chronic obstructive pulmonary disease; Alb, albumin; Hb, haemoglobin; Cr, creatinine; $\mathrm{BUN}$, blood urea nitrogen; AST, glutamic oxaloacetic transaminase; ALT, glutamic pyruvic transaminase; TBIL, total bilirubin; $d$, days.

internal fixation), the intraoperative blood loss and the duration of surgery did not influence the incidence of POP.

\section{Association Between Serum Albumin And Perioperative Parameters}

The demographic differences between patients with serum albumin $\geq 35 \mathrm{~g} / \mathrm{L}$ and patients with serum albumin $<35 \mathrm{~g} / \mathrm{L}$ (hypoalbuminaemia) are summarized in Table 2. The previous data showed that 125 patients were diagnosed with hypoalbuminaemia (17.36\%, 125 out of 720 patients), and the remaining 595 patients were placed in the normal serum albumin group. In terms of demographics, compared with patients with serum albumin $\geq 35 \mathrm{~g} / \mathrm{L}$, patients with serum albumin $<35 \mathrm{~g} / \mathrm{L}$ were older $(76.9 \pm 8.16$ vs $82.7 \pm 6.7$, $\mathrm{P}<0.0001)$. Unexpectedly, sex and BMI showed no significant differences between the two groups. Patients in the preoperative hypoalbuminaemia group tended to have higher rates of POP [32 (25.6\%) vs $22(3.69 \%), \mathrm{P}<0.0001]$ and higher rates of comorbidities, including arrhythmia [54 (43.0\%) vs $155(26.0 \%), \mathrm{P}<0.0001]$ and COPD [17 (13.6\%) vs $30(5.1 \%), \mathrm{P}<0.0001]$, than patients in the normal serum albumin group. Upon admission, patients in the preoperative hypoalbuminaemia group had lower haemoglobin $(113.1 \pm 17.9$ vs $122.8 \pm 17.25, \mathrm{P}<0.0001)$, but higher BUN (7.02 \pm 2.96 vs $6.16 \pm 2.81, \mathrm{P}=0.002)$ and serum creatinine levels $(72.9 \pm 30.6$ vs $67.57 \pm 20.84, \mathrm{P}=0.018)$ and a longer time from injury to surgery $(11.3 \pm 8.6$ vs $6.5 \pm 4.8$, $\mathrm{P}<0.0001)$ than patients in the normal serum albumin group. There was no difference in baseline characteristics, including the presence of coronary artery disease, hypertension, or prior stroke, smoking history, and serum ALT, AST, and TBIL levels, or in surgical parameters, including the 
Table 2 Association Between Serum Albumin And Perioperative Parameters

\begin{tabular}{|c|c|c|c|c|}
\hline Variable & Albumin $\geq 35 \mathrm{~g} / \mathrm{L} \mathrm{N}=595$ & Albumin $<35 \mathrm{~g} / \mathrm{L} \mathrm{N}=125$ & $\chi^{2}$ Or t-value & p value \\
\hline Age (years) & $76.9 \pm 8.6$ & $82.7 \pm 6.7$ & 5.759 & $<0.0001$ \\
\hline Sex, men/women & $233 / 362$ & $60 / 65$ & 3.345 & 0.067 \\
\hline BMI $\left(\mathrm{kg} / \mathrm{m}^{2}\right)$ & $22.32 \pm 1.97$ & $22.45 \pm 1.76$ & 0.712 & 0.476 \\
\hline POP (\%) & $22(3.69 \%)$ & $32(25.6 \%)$ & 71.43 & $<0.0001$ \\
\hline History of smoking (\%) & $83(13.9 \%)$ & 21 (I6.8\%) & 0.679 & $0.4 \mathrm{I}$ \\
\hline COPD $(\%)$ & $30(5.1 \%)$ & $17(13.6 \%)$ & 12.39 & $<0.0001$ \\
\hline Type 2 diabetes (\%) & $125(2 \mid .0 \%)$ & $23(18.4 \%)$ & 0.43 & 0.512 \\
\hline \multicolumn{5}{|l|}{ Circulatory diseases } \\
\hline Arrhythmia (\%) & I55 (26.0\%) & $54(43.0 \%)$ & 14.74 & $<0.0001$ \\
\hline Coronary artery disease (\%) & $262(44.0 \%)$ & $58(46.4 \%)$ & 0.234 & 0.628 \\
\hline Hypertension (\%) & 275 (46.2\%) & $65(0.52 \%)$ & 1.385 & 0.239 \\
\hline Prior stroke (\%) & $109(18.3 \%)$ & $20(16 \%)$ & 0.378 & 0.539 \\
\hline $\mathrm{Hb}(\mathrm{g} / \mathrm{L})$ & $122.8 \pm 17.25$ & $113.1 \pm 17.9$ & 5.655 & $<0.0001$ \\
\hline $\mathrm{Cr}(\mu \mathrm{mol} / \mathrm{L})$ & $67.57 \pm 20.84$ & $72.9 \pm 30.6$ & 2.370 & 0.018 \\
\hline BUN (mmol/L) & $6.16 \pm 2.81$ & $7.02 \pm 2.96$ & 3.054 & 0.002 \\
\hline ALT (U/L) & $18.92 \pm 21.63$ & $15.47 \pm 15.62$ & 1.689 & 0.092 \\
\hline AST (U/L) & $20.89 \pm 18.43$ & $17.57 \pm 6.97$ & 1.983 & 0.052 \\
\hline TBIL (umol/L) & $18.0 \pm 10.84$ & $18.84 \pm 13.96$ & 0.748 & 0.455 \\
\hline Surgical duration (min) & $81.9 \pm 26.6$ & $80.3 \pm 24.7$ & 0.661 & 0.508 \\
\hline Blood loss (mL) & $225 \pm 123.66$ & $204 \pm 114.86$ & 1.804 & 0.072 \\
\hline Time from injury to surgery (d) & $6.5 \pm 4.8$ & $11.3 \pm 8.6$ & 8.674 & $<0.0001$ \\
\hline Length of hospital stay (d) & $9.5 \pm 5.2$ & $10.5 \pm 3.8$ & 1.951 & 0.051 \\
\hline Procedure & & & 4.608 & 0.100 \\
\hline Total hip arthroplasty(\%) & I37 (23.0\%) & $18(14.4 \%)$ & & \\
\hline Intramedullary fixation (\%) & 77 (12.9\%) & $19(15.2 \%)$ & & \\
\hline Half hip replacement (\%) & $381(64.1 \%)$ & $88(70.4 \%)$ & & \\
\hline
\end{tabular}

Notes: The data are presented as percentage $(\mathrm{N})$ for categorical variables, mean \pm SD for continuous variables (normal distribution), median (IQR) for continuous variables (non-normal distribution).

Abbreviations: BMI, body mass index; POP, postoperative pneumonia; COPD, chronic obstructive pulmonary disease; Hb, haemoglobin; Cr, creatinine; BUN, blood urea nitrogen; AST, glutamic oxaloacetic transaminase; ALT, glutamic pyruvic transaminase; TBIL, total bilirubin.

length of hospital stay, surgical approaches, intraoperative blood loss and duration of surgery, between the hypoalbuminaemia group and the normal serum albumin group (Table 2).

\section{Predictive Factors For POP Using Univariate And Multivariable Analyses}

The results of univariate and multivariate analyses of preoperative and intraoperative potential risk factors are summarized in Table 3. From the univariate analysis, preoperative hypoalbuminaemia ( $\mathrm{OR}=8.962,95 \%$ CI: 4.991-16.093, $\mathrm{P}<0.0001)$, age (OR $=1.094,95 \%$ CI: 1.049-1.140, P<0.0001), COPD (OR $=6.714,95 \%$ CI: $3.327-13.548, \mathrm{P}<0.0001)$, history of smoking $(\mathrm{OR}=2.240,95 \% \mathrm{CI}: 1.172-4.282, \mathrm{P}=0.015)$, prior stroke (OR $=2.5,95 \%$ CI: $1.370-4.562, \mathrm{P}=0.003)$, admission haemoglobin $(\mathrm{OR}=0.979,95 \%$ CI: $0.967-0.991, \mathrm{P}=0.001)$, serum creatinine $(\mathrm{OR}=1.020,95 \%$ CI: $1.011-1.030, \mathrm{P}<0.0001)$ and time from injury to surgery $(\mathrm{OR}=1.109,95 \%$ CI: $1.073-1.147$,
$\mathrm{P}<0.0001)$ were found to be significantly associated with POP. Then, a multivariate analysis model was implemented to determine the independent predictive factors for POP. Preoperative hypoalbuminaemia $(\mathrm{OR}=5.187,95 \% \mathrm{CI}$ : 2.561-10.506, $\mathrm{P}<0.0001$ ), COPD (OR $=3.819,95 \%$ CI: $1.247-11.701, \mathrm{P}=0.019)$, prior stroke $(\mathrm{OR}=3.107,95 \%$ CI: $1.470-6.568, \mathrm{P}=0.003$ ) and time from injury to surgery ( $\mathrm{OR}=1.076,95 \%$ CI: $1.034-1.119, \mathrm{P}<0.0001)$ were maintained in the model after adjustment, indicating that they were independent risk factors for POP. Patients with preoperative hypoalbuminaemia were at 5.187 times greater risk of POP than patients with normal preoperative albumin levels (Table 3).

\section{Discussion}

In the current study, the incidence of POP after femoral neck fracture surgery in the geriatric population was $7.5 \%$. 
Table 3 Predictive Factors For POP Using Univariate And Multivariable Analyses

\begin{tabular}{|c|c|c|c|c|}
\hline \multirow[t]{2}{*}{ Variables } & \multicolumn{2}{|l|}{ Univariate } & \multicolumn{2}{|l|}{ Multivariable } \\
\hline & OR $(95 \% \mathrm{CI})$ & $\mathbf{P}$ & OR $(95 \% \mathrm{CI})$ & $\mathbf{P}$ \\
\hline Age (years) & $1.094(1.049-1.140)$ & $<0.0001$ & $1.030(0.974-1.089)$ & 0.304 \\
\hline Sex (female) & $1.159(0.653-2.057)$ & 0.614 & & \\
\hline BMI $\left(\mathrm{kg} / \mathrm{m}^{2}\right)$ & $0.917(0.800-1.052)$ & 0.217 & & \\
\hline History of smoking & $2.240(1.172-4.282)$ & 0.015 & $1.133(0.409-3.141)$ & 0.810 \\
\hline COPD & $6.714(3.327-13.548)$ & $<0.0001$ & $3.819(1.247-11.701)$ & 0.019 \\
\hline Type 2 diabetes & $1.703(0.921-3.3149)$ & 0.089 & $2.046(0.984-4.258)$ & 0.055 \\
\hline Arrhythmia & I.485(0.833-2.645) & 0.180 & & \\
\hline Coronary artery disease & I. I75(0.674-2.047) & 0.569 & & \\
\hline Hypertension & $1.222(0.70 \mathrm{I}-2.129)$ & 0.479 & & \\
\hline Prior stroke & $2.5(1.370-4.562)$ & 0.003 & $3.107(1.470-6.568)$ & 0.003 \\
\hline Hypoalbuminaemia & $8.962(4.991-16.093)$ & $<0.0001$ & $5.187(2.56 \mathrm{I}-10.506)$ & $<0.0001$ \\
\hline $\mathrm{Hb}$ & $0.979(0.967-0.991)$ & 0.001 & $0.989(0.972-1.005)$ & 0.175 \\
\hline $\mathrm{Cr}$ & $1.020(1.011-1.030)$ & $<0.0001$ & $1.016(0.998-1.003)$ & 0.075 \\
\hline BUN & $1.030(0.946-1.112)$ & 0.499 & & \\
\hline ALT & $1.006(0.996-1.0016)$ & 0.261 & & \\
\hline AST & $0.984(0.953-1.017)$ & 0.339 & & \\
\hline TBIL & $0.988(0.961-1.016)$ & 0.386 & & \\
\hline Surgical duration time & $0.993(0.981-1.005)$ & 0.240 & & \\
\hline Blood loss & $1.000(0.997-1.002)$ & 0.858 & & \\
\hline Time from injury to surgery & I.109(1.073-I.I47) & $<0.0001$ & $1.076(1.034-1.119)$ & $<0.0001$ \\
\hline Surgical approaches & $1.399(0.576-3.398)$ & 0.347 & & \\
\hline
\end{tabular}

Abbreviations: BMI, body mass index; COPD, chronic obstructive pulmonary disease; Alb, albumin; Hb, haemoglobin; Cr, creatinine; BUN, blood urea nitrogen; AST, glutamic oxaloacetic transaminase; ALT, glutamic pyruvic transaminase; TBIL, total bilirubin.

The rates of POP were significantly higher in the hypoalbuminaemia group than in the normal serum albumin group. In addition, patients with POP had significantly longer hospital stays than patients without POP group. The binary logistic regression analysis revealed that preoperative hypoalbuminaemia was independently associated with a 5.187-fold increase in the rate of POP after femoral neck fracture in the geriatric population. The present study also demonstrated that COPD, prior stroke and time from injury to surgery were predominant and independent risk factors for POP after femoral neck fracture in the geriatric population.

Our results showed that hypoalbuminaemia was an independent risk factor for predicting POP after femoral neck fracture surgery in the geriatric population. In this cohort, hypoalbuminaemia was present in $17.36 \%$ of patients with femoral neck fracture on admission to the hospital. The incidence of hypoalbuminaemia observed in our cohort of patients is similar to what Kamath $\mathrm{A}$, et $\mathrm{al}^{20}$ reported in their study. They examined 4551 consecutive patients admitted for revision total knee arthroplasty and found hypoalbuminaemia in $16.1 \%$ of patients. Perioperative nutritional status is an important factor correlated with postoperative outcomes, and hypoalbuminaemia is a predictor of postoperative morbidity. $^{21}$ Previous studies have shown that nutritional status plays an important role in inpatient recovery from surgery and may be responsible for the incidence of perioperative morbidity after surgery. ${ }^{22}$ The serum albumin level indicates nutritional status, is regarded as a negative acutephase protein and is produced in the liver at a level of 12-25 $\mathrm{g} / \mathrm{d}$; it is the most abundant blood plasma protein and has a half-life of 20 days. The serum albumin concentration is affected by multiple factors, including nutritional status, liver failure, malignancy, surgical trauma, bacterial infection, inflammation, acute and chronic comorbidities (e.g., diabetes) and other conditions. ${ }^{15}$ In the present study, all our patients experienced trauma. It is known that the stress response to injury is a factor that contributes to low preoperative serum levels. $^{23,24}$ These low serum levels may impair the ability of the immune system to fight infection. ${ }^{24}$

Our results are consistent with several previous studies that researched the relationship between preoperative hypoalbuminaemia and POP. In the present study, the rates of POP were much higher in the hypoalbuminaemia group than in the normal serum albumin group. Several studies have indicated that preoperative hypoalbuminaemia is a well-documented 
risk factor for postoperative complications in various types of surgery. ${ }^{17,25}$ Kamath $\mathrm{A}$ et $\mathrm{al}^{20}$ reported that hypoalbuminaemia was independently associated with an increased risk of developing deep organ surgical-site infections, surgical-site infections, urinary traction infections, pneumonia and sepsis after revision total knee arthroplasty. Rungsakulkij $\mathrm{N}$ et al ${ }^{15}$ reported that perioperative albumin was an important factor associated with serious complications following pancreaticoduodenectomy, include POP. This evidence was also supported by Munteanu A et $\mathrm{al}^{26}$ who reported that serum albumin levels, checked both before and after surgery, were of great value in helping predict a series of postoperative complications in gastric cancer surgery. Though their studies were not focused on femoral neck fracture patients, their results were quite similar to ours regarding preoperative hypoalbuminaemia in predicting postoperative complications.

Measuring the level of serum albumin is still a simple and standard method to assess protein energy malnutrition in patients with hip fractures. ${ }^{27}$ Such information provides significant prognostic information and can help identify those who may benefit from nutritional supplements. ${ }^{27}$ An increase in protein and energy intake prior to surgery can prevent malnutrition, improve recovery from surgery, reduce postoperative complications, shorten hospitalization and decrease the incidence of postoperative morbidity in patients after surgery. ${ }^{28}$ Espauella et al $^{29}$ reported in a randomized, double-blinded placebo-controlled trial that patients with hip fractures who had hypoalbuminaemia and received nutritional supplementation had lower rates of postoperative complications compared to the control group. Similarly, Bohl $\mathrm{D}$ et $\mathrm{al}^{30}$ reported that among patients admitted for abdominal surgery in a multicentre prospective cohort study, the total complication rate was lower in the group that received nutritional supplementation than in the control group.

Hypoalbuminaemia has also been found to be an independent predictor of prolonged hospital length of stay in hip fracture patients. ${ }^{29}$ However, in our study, we did not find a relationship between hypoalbuminaemia and the length of hospital stay, which is not consistent with other studies. This could be explained by the fact that malnutrition is associated with lower immune responses, older age, muscle wasting, poor cardiac-pulmonary function, and cognitive and functional premorbid status. These comorbidities may further compound the effects of nutritional status and postoperative outcomes. On the other hand, our study is a small sample study, and if we expand the sample size in future studies, we may have different results.
Despite this inconsistency, patients who were diagnosed with POP had a longer hospital stays than patients without POP. This increase in the length of stay is of momentous clinical and economic importance, and this result is consistent with two other studies of hip fracture and total joint arthroplasty patients. ${ }^{13,30}$ Older populations usually have severe comorbidities, and they may be high-risk surgical candidates. ${ }^{10}$ Consistent with previous reports, our study also found that patients with POP had more comorbidities. COPD and prior stroke were independent prognostic indicators for POP in elderly patients with femoral neck fractures. Previous studies have shown the advantage of surgery within $48 \mathrm{hrs}$ of injury, supporting the observation that hip fracture surgeries within $48 \mathrm{hrs}$ of admission have better outcomes. ${ }^{31}$ Researchers have suggested that a prolonged time from injury to surgery is associated with an increased incidence of pneumonia and other complications after surgery for a femoral neck fracture. ${ }^{32}$ Similarly, our study demonstrated a higher incidence of POP among patients with femoral neck fractures who had longer times from injury to surgery. The incidence of POP in patients with longer times from injury to surgery was 1.076 times that of POP in patients with shorter times from injury to surgery.

There are some limitations to our study. First, it was conducted retrospectively at a single institution, which may limit its applicability. Causality between clinical preoperative hypoalbuminaemia and POP risk cannot be determined. Nonetheless, our analysis identified preoperative hypoalbuminaemia as an indicator of high risk for POP. Second, in the current study, the main endpoint was the occurrence of POP, and other perioperative or postoperative morbidities were not explored. Additionally, there may be other risk factors affecting the development of POP that we did not take into account during this retrospective study. For example, this study lacks assessment of patient cognitive and functional status, which also affects the occurrence of POP. In addition, information was collected only from the 30th postoperative day, potentially missing cases of pneumonia that occurred after this period. Third, we did not examine other markers of malnutrition, such as total lymphocyte count, vitamin D levels, prealbumin levels, or transferrin levels. Therefore, malnutrition was only based on hypoalbuminaemia, which could mean that the real prevalence of malnutrition was underestimated in this study. In contrast, we focused on only preoperative albumin levels, and we did not study postoperative or dynamic changes in serum albumin 
during the perioperative period and their relationship with POP. These factors should also be considered in future research. Further, the number of patients included in the current study was not large enough. Therefore, the present findings require further validation by large scale, prospective, randomized, multicentre parallel control trials.

\section{Conclusions}

In conclusion, the current study revealed that among a geriatric population admitted for femoral neck fracture surgery, preoperative hypoalbuminaemia was the leading predictor of POP, followed by COPD, prior stroke and the time from injury to surgery. In addition, patients with POP had significantly longer hospital stays than patients without POP. Thus, patients who undergo femoral neck fracture surgery should have their serum albumin levels measured routinely on admission, and individuals with preoperative hypoalbuminaemia should receive increased monitoring and intensive perioperative care. Further studies are needed to assess whether nutritional support can decrease the risk of POP in patients with hypoalbuminaemia.

\section{Acknowledgments}

We kindly thank all participants for their participation in our study. And we thank AJE for editing a draft of this manuscript. This work was supported by Shaanxi Natural Science Foundation of China (Number 2019JQ-536).

\section{Disclosure}

The authors report no conflicts of interest in this work.

\section{References}

1. Ji C, Zhu Y, Liu S, et al. Incidence and risk of surgical site infection after adult femoral neck fractures treated by surgery. Medicine. 2019;98(11):e14882. doi:10.1097/MD.0000000000014882

2. Bergeron E, Moore L, Fournier K, Gravel C, Lavoie A. Patients with isolated hip fracture must be considered for surgery irrespectively of their age, comorbidity status and provenance: a statement applicable even to nonagenarians. Arch Orthop Trauma Surg. 2009;129 (11):1549-1555. doi:10.1007/s00402-009-0888-7

3. Lv H, Yin P, Long A, et al. Clinical characteristics and risk factors of postoperative pneumonia after hip fracture surgery: a prospective cohort study. Osteoporosis Int. 2016;27(10):3001-3009. doi:10.1007/s00198016-3624-5

4. Lo IL, Siu CW, Tse HF, Lau TW, Leung F, Wong M. Pre-operative pulmonary assessment for patients with hip fracture. Osteoporos Int. 2010;21(Suppl 4):S579-S586. doi:10.1007/s00198-010-1427-7

5. Bohl DD, Sershon RA, Saltzman BM, Darrith B, Della Valle CJ. Incidence, risk factors, and clinical implications of pneumonia after surgery for geriatric hip fracture. J Arthroplasty. 2018;33(5):15521556. doi: $10.1016 /$ j.arth.2017.11.068
6. Hu F, Jiang C, Shen J, Tang P, Wang Y. Preoperative predictors for mortality following hip fracture surgery: a systematic review and metaanalysis. Injury. 2012;43(6):676-685. doi:10.1016/j.injury.2011.05.017

7. Hattori K, Matsuda T, Takagi Y, et al. Preoperative six-minute walk distance is associated with pneumonia after lung resection. Interact Cardiov Th. 2018;26(2):277-283. doi:10.1093/icvts/ivx310

8. López-de-Andrés A, Perez-Farinos N, de Miguel-díez J, et al. Type 2 diabetes and postoperative pneumonia: an observational, populationbased study using the Spanish Hospital discharge database, 2001-2015. PLoS One. 2019;14(2):e211230. doi:10.1371/journal.pone.0211230

9. Jung J, Moon SM, Jang HC, et al. Incidence and risk factors of postoperative pneumonia following cancer surgery in adult patients with selected solid cancer: results of "Cancer POP" study. Cancer Med. 2018;7(1):261-269. doi:10.1002/cam4.1259

10. Chughtai M, Gwam CU, Mohamed N, et al. The epidemiology and risk factors for postoperative pneumonia. J Clin Med Res. 2017;9 (6):466-475. doi:10.14740/jocmr3002w

11. Wei R, Dong W, Shen H, et al. Predictive effects of lung function test on postoperative pneumonia in squamous esophageal cancer. Sci RepUK. 2016;6:1.

12. Aldecoa C, Bettelli G, Bilotta F, et al. European society of anaesthesiology evidence-based and consensus-based guideline on postoperative delirium. Eur J Anaesthesiol. 2017;34(4):192-214. doi:10.1097/ EJA.0000000000000594

13. Aldebeyan S, Nooh A, Aoude A, Weber MH, Harvey EJ. Hypoalbuminaemia - a marker of malnutrition and predictor of postoperative complications and mortality after hip fractures. Injury. 2017;48(2):436-440. doi:10.1016/j.injury.2016.12.016

14. Bohl DD, Shen MR, Hannon CP, Fillingham YA, Darrith B, Della Valle CJ. Serum albumin predicts survival and postoperative course following surgery for geriatric hip fracture. $J$ Bone Joint Surg. 2017;99(24):2110-2118. doi:10.2106/JBJS.16.01620

15. Rungsakulkij N, Tangtawee P, Suragul W, Muangkaew P, Mingphruedhi S, Aeesoa S. Correlation of serum albumin and prognostic nutritional index with outcomes following pancreaticoduodenectomy. World J Clin Cases. 2019;7(1):28-38.

16. Wierdak M, Pisarska M, Kuśnierz-Cabala B, et al. Changes in plasma albumin levels in early detection of infectious complications after laparoscopic colorectal cancer surgery with ERAS protocol. Surg Endosc. 2018;32(7):3225-3233. doi:10.1007/s00464-018-6040-4

17. Li P, Li J, Lai Y, et al. Perioperative changes of serum albumin are a predictor of postoperative pulmonary complications in lung cancer patients: a retrospective cohort study. J Thorac Dis. 2018;10 (10):5755-5763. doi:10.21037/jtd

18. Yi PH, Frank RM, Vann E, Sonn KA, Moric M, Della VC. Is potential malnutrition associated with septic failure and acute infection after revision total joint arthroplasty? Clin Orthop Relat Res. 2015;473(1):175-182. doi:10.1007/s11999-014-3685-8

19. Kieninger AN, Lipsett PA. Hospital-acquired pneumonia: pathophysiology, diagnosis, and treatment. Surg Clin North Am. 2009;89 (2):439-461. doi:10.1016/j.suc.2008.11.001

20. Kamath A, Nelson C, Elkassabany N, Guo Z, Liu J. Low albumin is a risk factor for complications after revision total knee arthroplasty. $J$ Knee Surg. 2017;30(3):269-275. doi:10.1055/s-0036-1584575

21. Costa MD, Vieira DMC, Amorim AC, Cipriano TDO, Dos SA. Association between nutritional status, inflammatory condition, and prognostic indexes with postoperative complications and clinical outcome of patients with gastrointestinal neoplasia. Nutr Cancer. 2016;68(7):1108-1114. doi:10.1080/01635581.2016.1206578

22. Okada S, Shimada J, Kato D, Tsunezuka H, Teramukai S, Inoue M. Clinical significance of prognostic nutritional index after surgical treatment in lung cancer. Ann Thorac Surg. 2017;104(1):296-302. doi:10.1016/j.athoracsur.2017.01.085

23. Neel DR, McClave S, Martindale R. Hypoalbuminaemia in the perioperative period: clinical significance and management options. Best Pract Res Clin Anaesthesiol. 2011;25(3):395-400. doi:10.1016/j.bpa.2011.07.006 
24. Weimann A, Braga M, Carli F, et al. ESPEN guideline: clinical nutrition in surgery. Clin Nutr. 2017;36(3):623-650.

25. Ataseven B, du Bois A, Reinthaller A, et al. Pre-operative serum albumin is associated with post-operative complication rate and overall survival in patients with epithelial ovarian cancer undergoing cytoreductive surgery. Gynecol Oncol. 2015;138(3):560-565. doi:10.1016/j.ygyno.2015.07.005

26. Munteanu A, Munteanu D, Tigan S, Bartos A, Iancu C. How do surgical stress and low perioperative serum protein and albumin impact upon short term morbidity and mortality in gastric cancer surgery? Med Pharm Rep. 2017;90(1):71-85. doi:10.15386/cjmed-674

27. O’Daly BJ, Walsh JC, Quinlan JF, et al. Serum albumin and total lymphocyte count as predictors of outcome in hip fractures. Clin Nutr. 2010;29(1):89-93. doi:10.1016/j.clnu.2009.07.007

28. Hoekstra JC, Goosen JH, de Wolf GS, Verheyen CC. Effectiveness of multidisciplinary nutritional care on nutritional intake, nutritional status and quality of life in patients with hip fractures: a controlled prospective cohort study. Clin Nutr. 2011;30(4):455-461. doi:10.1016/j.clnu.2011.01.011
29. Espaulella J, Guyer H, Diaz-Escriu F, Mellado-Navas JA, Castells M, Pladevall M. Nutritional supplementation of elderly hip fracture patients. A randomized, double-blind, placebo-controlled trial. Age Ageing. 2000;29(5):425-431. doi:10.1093/ageing/29.5.425

30. Bohl DD, Shen MR, Kayupov E, Della Valle CJ. Hypoalbuminemia independently predicts surgical site infection, pneumonia, length of stay, and readmission after total joint arthroplasty. $J$ Arthroplasty. 2016;31(1):15-21. doi:10.1016/j.arth.2015.08.028

31. Roberts KC, Brox WT, Jevsevar DS, Sevarino K. Management of hip fractures in the elderly. J Am Acad Orthop Surg. 2015;23(2):131137. doi:10.5435/JAAOS-D-14-00432

32. Westberg M, Snorrason F, Frihagen F. Preoperative waiting time increased the risk of periprosthetic infection in patients with femoral neck fracture. Acta Orthop. 2013;84(2):124-129. doi:10.3109/ 17453674.2013.775044
Clinical Interventions in Aging

\section{Publish your work in this journal}

Clinical Interventions in Aging is an international, peer-reviewed journal focusing on evidence-based reports on the value or lack thereof of treatments intended to prevent or delay the onset of maladaptive correlates of aging in human beings. This journal is indexed on PubMed Central, MedLine, CAS, Scopus and the Elsevier

\section{Dovepress}

Bibliographic databases. The manuscript management system is completely online and includes a very quick and fair peer-review system, which is all easy to use. Visit http://www.dovepress.com/ testimonials.php to read real quotes from published authors. 